\title{
Patterns of disease activity in multiple sclerosis: clinical and magnetic resonance imaging study
}

\author{
Alan J Thompson, Allan G Kermode, D G MacManus, B E Kendall, D P E Kingsley, I F Moseley, \\ W I McDonald
}

\begin{abstract}
Objective-To compare the abnormalities shown by magnetic resonance imaging of the brain in three clinically distinct groups of patients with multiple sclerosis, and to correlate the extent of abnormality with the degree of clinical disability in the three groups.

Design-All patients underwent magnetic resonance imaging and full neurological examination, and their disability was scored according to the expanded Kurtzke disability state scale.

Setting - National Hospital for Nervous Diseases (Multiple Sclerosis NMR Research Group).

Patients - Three groups of patients with confirmed multiple sclerosis were studied: 12 patients with minimal disability despite a long ( $>10$ years) duration of illness (benign multiple sclerosis), 16 who had developed progressive disability after a relapsing and remitting course (secondary progressive multiple sclerosis), and 13 who had had progressive disability from the onset of the disease (primary progressive
\end{abstract} multiple sclerosis).

Main outcome measures-Number and size of lesions in 17 anatomically defined sites; total lesion load, estimated with an arbitrary scoring system weighted for the size of lesions; and disability score.

Results-Magnetic resonance imaging showed that all 41 patients had abnormalities. These were extensive in the groups with secondary progressive and benign disease compared with the group with primary progressive disease. The lesions in the patients with secondary progressive disease were larger and more confluent than those in the two other groups $(p=0 \cdot 007)$. Most lesions $(85 \%)$ in the patients with primary progressive disease were under $5 \mathrm{~mm}$ in diameter; this percentage was higher than that in the two other groups $(p=0.032)$. Consequently the patients with primary progressive disease had the lowest mean lesion load (36.7); that in the patients with benign disease was 52.7 and that in the patients with secondary progressive disease $64.6(p=0.05)$. No correlation existed between disability and total lesion load. The distribution of brain lesions and of detectable lesions of the spinal cord, and the frequency of cortical atrophy, were similar in all groups.

Conclusions-No relation was found between the degree of clinical disability and the extent of abnormality shown by magnetic resonance imaging: patients with clinically benign disease often had extensive abnormalities and those with primary progressive disease had surprisingly few lesions. Though magnetic resonance imaging increases knowledge of the disease process in multiple sclerosis and is invaluable in diagnosis, it is not helpful in predicting disability in individual patients.

\section{Introduction}

The variation in the clinical presentation of multiple sclerosis and the course of the disease is well recognised. ${ }^{1.3}$ In most patients the condition has a relapsing and remitting course at first, but after a variable period progressive disability occurs, often with superimposed relapses (secondary progressive disease). In about $30 \%$ of patients the disease follows a fairly benign course and appreciable disability has not developed 10 to 15 years after its onset. ${ }^{45}$ Patients in whom the disease is destined to have a benign outcome cannot be identified at presentation, though they are more likely to present with purely sensory symptoms and to have a lengthy first remission. ${ }^{5}$ Their main distinguishing features, however, are their continuing capacity to recover from relapses and the fact that they do not develop progressive disability. There is also a clinically distinct group of patients who never have relapses or remissions but whose disability is progressive from the onset (primary progressive disease). These patients constitute between $7 \%$ and $13 \%$ of people with multiple sclerosis ${ }^{36}$ and tend to be older at onset than the others. They often present with a spastic paraparesis, which then deteriorates slowly. The factors that influence the development of disability in multiple sclerosis are not clearly understood, but the site and extent of the area of demyelination, the presence of wallerian degeneration, and the capacity for remyelination are all likely to be relevant.?

Using magnetic resonance imaging it is now possible to show lesions in the brain and spinal cord of patients with multiple sclerosis. Abnormalities are seen in over $95 \%$ of patients with clinically definite disease and have been correlated with plaques found at postmortem examination. ${ }^{89}$ Thus it seems reasonable to use the extent of abnormality shown by magnetic resonance imaging as an index of the extent of pathological change during life. As doctors sometimes ask whether magnetic resonance imaging can give a guide to prognosis in patients with multiple sclerosis we examined scans of patients in the three groups described above to see, firstly, if the pattern and extent of abnormality shown by magnetic resonance imaging could help to explain the wide variation in clinical course between the three groups and, secondly, if the extent of abnormality shown by imaging correlated with clinical disability.

\section{Patients and methods}

We examined 41 patients with multiple sclerosis confirmed clinically or by laboratory tests ${ }^{10}$ who were taking part in a serial study. Twelve had benign, 16 secondary progressive, and 13 primary progressive disease. All patients had IgG oligoclonal bands in cerebrospinal fluid as shown by electrophoresis.

The disability of the patients was assessed by one of us (AJT) at the time of each nuclear magnetic resonance scan and was given a score according to the expanded
Correspondence to: $\mathrm{Dr}$ Thompson.

BrMed f 1990;300:631-4
0 MARCH 1990 
Kurtzke disability state scale. ${ }^{11}$ This is a 10 point scale that takes account of neurological deficit in all the functional systems but is heavily weighted towards loss of mobility. Patients with a score $\leqslant 3$ have minimal disability, can walk unaided, and lead an independent existence. A score of 6 indicates that help is required to walk even short distances, and a score $\geqslant 7$ implies that the patient is essentially confined to a wheelchair. By definition patients with benign multiple sclerosis had a disability score $\leqslant 3$ and had had the disease for more than 10 years. Patients with secondary progressive multiple sclerosis had had an initial relapsing and remitting course but were in a progressive phase and had reported definite deterioration during the six months before the study was started. Patients with primary progressive disease had always had progressive disease, with no suggestion of either a relapse or a remission; they also had evidence (clinical or investigative) that a second anatomically distinct area of the central nervous system had been affected after the first.

Relapse was defined as the occurrence of new symptoms and signs or a deterioration in existing symptoms and signs of at least 24 hours' duration in the absence of fever.

$\mathrm{T}_{2}$ weighted magnetic resonance imaging of the brain was performed with a $0.5 \mathrm{~T}$ superconducting

TABLE I-Clinical features of three groups of patients with multiple sclerosis. Values are means (SD)

\begin{tabular}{|c|c|c|c|}
\hline & \multicolumn{3}{|c|}{ Classification of multiple sclerosis } \\
\hline & $\begin{array}{l}\text { Primary progressive } \\
\qquad(\mathrm{n}=13)\end{array}$ & $\begin{array}{l}\text { Secondary progressive } \\
\qquad(n-16)\end{array}$ & $\begin{array}{l}\text { Benign } \\
(n=12)\end{array}$ \\
\hline \multirow{3}{*}{$\begin{array}{l}\text { Age (years) } \\
\text { Duration of disease (years) } \\
\text { Score on expanded Kurtzke } \\
\text { disability state scale }\end{array}$} & $43 \cdot 0(10 \cdot 7)$ & $38 \cdot 1(9 \cdot 3)$ & $42 \cdot 0(8 \cdot 7)$ \\
\hline & $6 \cdot 8(4 \cdot 0)$ & $9 \cdot 3(4 \cdot 8)$ & $18 \cdot 1(6 \cdot 6)$ \\
\hline & $4.5(1.3)$ & $5 \cdot 3(1 \cdot 0)$ & $2 \cdot 2(0 \cdot 6)$ \\
\hline
\end{tabular}

TABLE II-Mean (SD) numbers of abnormalities shown by magnetic resonance imaging of brain in three groups of patients with multiple sclerosis

\begin{tabular}{lcccc}
\hline & \multicolumn{3}{c}{ Classification of multiple sclerosis } & \\
\cline { 2 - 4 } & $\begin{array}{c}\text { Primary progressive } \\
(\mathrm{n}=13)\end{array}$ & $\begin{array}{c}\text { Secondary progressive } \\
(\mathrm{n}=16)\end{array}$ & $\begin{array}{c}\text { Benign } \\
(\mathrm{n}=12)\end{array}$ & p Value \\
\hline $\begin{array}{l}\text { No of lesionsipatient } \\
\text { No of confluent lesions }\end{array}$ & $30 \cdot 5(18 \cdot 0)$ & $44 \cdot 3(21 \cdot 7)$ & $40 \cdot 7(20 \cdot 3)$ & $0 \cdot 15$ \\
$\begin{array}{l}>5 \mathrm{~mm} \text { diameter } \\
\text { \% Of lesions }<5 \mathrm{~mm}\end{array}$ & $1 \cdot 3(2 \cdot 5)$ & $5 \cdot 0(4 \cdot 0)$ & $1 \cdot 5(2 \cdot 5)$ & $0 \cdot 007$ \\
$\begin{array}{l}\text { diameter } \\
\text { Lesion load }\end{array}$ & $85(17)$ & $59(15)$ & $75(19)$ & $0 \cdot 032$ \\
\hline
\end{tabular}

^Arbitrary score; see text for details. machine (Picker; spin echo time $2000 /(1), 5 \mathrm{~mm}$ contiguous axial slices). The scanning plane was deter mined with oblique pilots and internal landmarks. which ensured that the positions of the slices werc similar for each patient. Spin echo time ${ }_{500 / 40}(\mathrm{~T}$ weighted) and spin echo time ${ }_{1500 / 80}\left(\mathrm{~T}_{2}\right.$ weighted $5 \mathrm{~mm}$ parasagittal slices of the cervical and thoracic cord were also obtained for most of the patients. Scans were examined by two of us (DPEK and BEK), who. were unaware of the disease pattern. Lesions werc counted and their diameters measured for 17 anatomically defined locations. An arbitrary scoring system weighted for the size of the lesions was used to estimate the total lesion load: each lesion of diameter $<5 \mathrm{~mm}$ scored 1; 5-10 mm, 2; and $>10 \mathrm{~mm}, 3$. Periventricular confluent lesions, which were often extensive, were measured at their maximum diameter in the coronal plane only and scored one extra point over discrete lesions. Results were analysed with Kruskal-Wallis one way analysis of variance.

\section{Results}

The mean age of each group was similar, but by definition patients with benign disease had had the disease longer and had less disability than the two groups with progressive disease. Disability and duration of disease were similar in the two groups with progressive disease (table I).

Magnetic resonance imaging showed abormalities in all 41 patients (table II). Extensive abnormalities were seen in the groups with secondary progressive and benign disease (fig 1 (left and centre)) and only minor abnormalities in the group with primary progressive disease (fig 1 (right)). Patients with secondary progressive multiple sclerosis had most lesions but only slightly more than those with benign disease. The lesions in those with secondary progressive disease were larger and more confluent than those in the two other groups $(p=0.007)$. Patients with primary progressive disease had few lesions, and most of these $(85 \%)$ were under $5 \mathrm{~mm}$ in size; this was a higher percentage than in either of the other groups $(p=$ 0.032 ). As a consequence the patients with primary progressive disease had the lowest mean lesion load (36.7) compared with the patients with benign $(52 \cdot 7)$ and secondary progressive $(64 \cdot 6)$ disease $(\mathrm{p}=0.05)$. There was no correlation between disability and total lesion load (fig 2). The distribution of lesion load was similar in all three groups (table III).
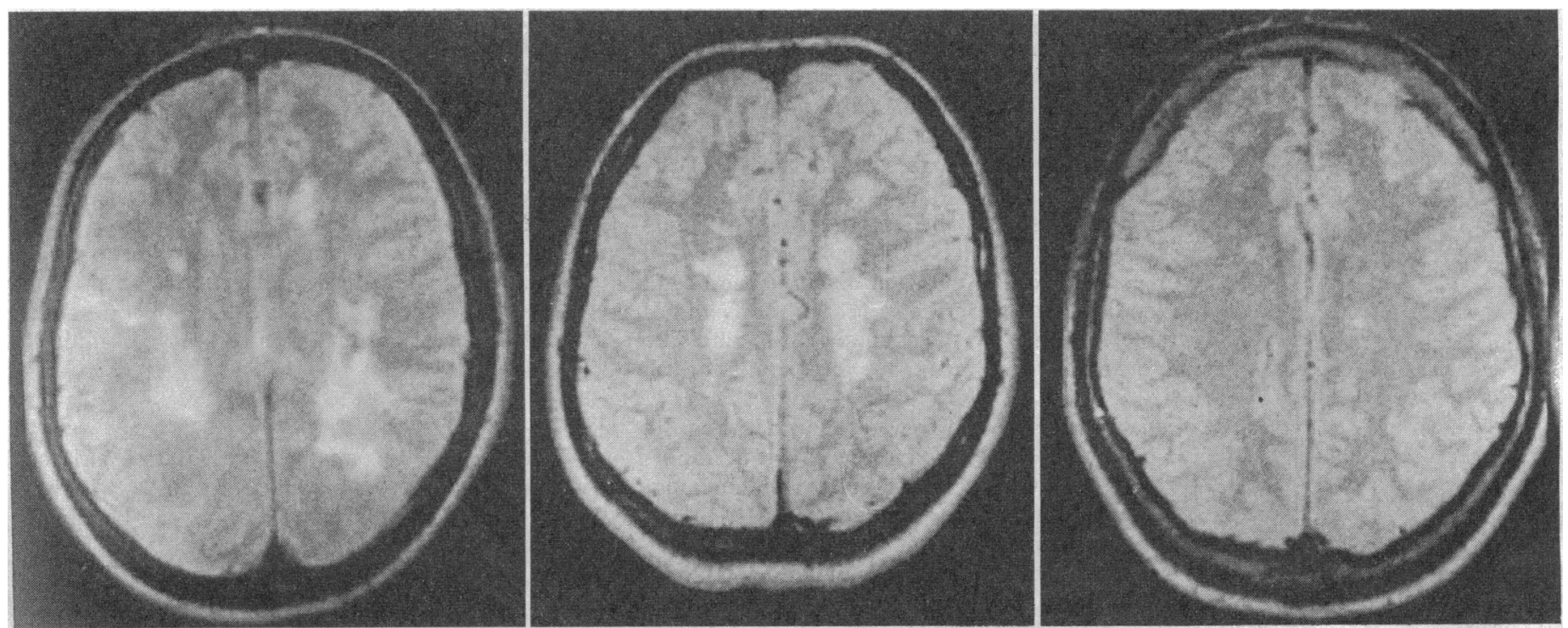

FIG 1-T, weighted magnetic resonance images ( $S E_{2}$, of $5 \cdot 5$, showing large confluent lesions; (centre) 49 year old woman with benign multiple sclerosis of 19 years' duration and disability score of $2 \cdot 0$, showing large confluent lesions; ( right) 44 vear old man with primary progressive multiple sclerosis of 11 years' duration and disability score of 6.0 , showing few small lesions 


\begin{tabular}{lccc} 
& \multicolumn{3}{c}{ Classification of multiple sclerosis } \\
\cline { 2 - 4 } & Primary progressive & Secondary progressive & Benign \\
\hline Periventricular & 18.5 & $38 \cdot 3$ & $26 \cdot 4$ \\
Discrete cerebral white matter & $15 \cdot 5$ & $24 \cdot 4$ & 26.7 \\
$\quad$ Internal capsule & $1 \cdot 4$ & $2 \cdot 1$ & $2 \cdot 6$ \\
Brain stem & $3 \cdot 5$ & 3.9 & $4 \cdot 0$ \\
Cerebellum & $1 \cdot 0$ & $2 \cdot 4$ & $1 \cdot 4$ \\
\hline Total & 38.5 & $69 \cdot 0$ & $58 \cdot 5$ \\
\hline
\end{tabular}

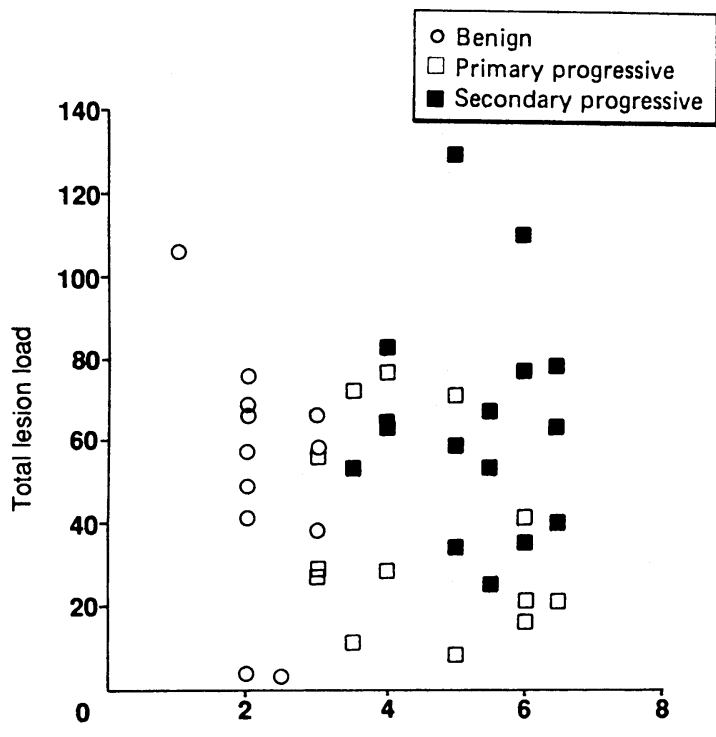

Score on expanded Kurtzke disability state scale

FIG 2-Scatter diagram of lesion load against disability measured on expanded Kurtzke disability state scale in patients with benign, primary progressive, and secondary progressive multiple sclerosis

Cortical atrophy was rare and was seen in only one patient with primary progressive disease, one with benign disease, and five with secondary progressive disease. Abnormalities of the spinal cord were seen in three of nine patients with primary progressive multiple sclerosis, seven of the 16 patients with secondary progressive disease, and five of 11 patients with benign disease.

\section{Discussion}

The most striking finding in our study was the disparity between the extent of the patients' disability and the extent of abnormality shown by magnetic resonance imaging: the minimally disabled group with benign disease had considerably more lesions than the group with primary progressive disease.

One possible explanation for the disparity between lesion load and disability in benign multiple sclerosis is that a high proportion of the lesions are clinically silent. It is now established from serial studies using magnetic resonance imaging that patients with multiple sclerosis have significantly more new lesions than clinical relapses. ${ }^{12-14} \mathrm{~A}$ comparison of the patients with benign disease and the more disabled patients with secondary progressive disease in our study, however, showed that there was little difference in the distribution of lesions. As already described, ${ }^{15}$ lesions in the patients with secondary progressive disease were larger and more confluent but tended to be in the periventricular areas, damage to which does not result in obvious neurological deficit. Koopmans et al found more infratentorial lesions in patients with secondary progressive disease than in patients with benign disease, ${ }^{15}$ but this was not the case in our patients. Importantly, they found that many infratentorial lesions in the patients with benign disease had never caused symptoms.

As well as the site of the lesions their nature and activity need to be considered. In multiple sclerosis an abnormal signal on magnetic resonance imaging derives in part from cellular changes in the lesion and in part from oedema. Oedema is often more extensive than the lesion. ${ }^{16}$ As clinical deficit is determined by damage to nerve fibres a poor correlation at any one time between the area of the lesion and disability is not surprising. A further factor influencing the severity of deficit is the efficiency of the recovery mechanisms that operate after conduction is blocked in damaged nerve fibres. These complex issues are discussed elsewhere.

The second major finding in this study was the relative lack of lesions found by magnetic resonance imaging in the patients with primary progressive disease compared with those with secondary progressive disease despite similar disability; lesions in those with primary progressive disease were fewer and smaller, even in severely disabled patients. The site of the cerebral lesions cannot explain this as the patients with primary progressive multiple sclerosis tended to have fewer lesions in such potentially disabling areas as the internal capsule, brain stem, and cerebellum (table III). Similarly, there was no significant difference in the frequency of spinal cord lesions between the two groups, although the trend was suggestive of fewer lesions in the patients with primary progressive disease. Results from scanning the spinal cord, however, are not yet as satisfactory as those from scanning the brain, and hence the error in assessing lesion load in the spinal cord is correspondingly greater and differences may be missed.

Most lesions in the patients with primary progressive disease tended to be small, and many may have been below the resolution of the scanner. Conduction can be blocked by as little as a $1 \mathrm{~mm}$ length of demyelination, so that large numbers of small lesions, not visible by magnetic resonance imaging, could produce a severe clinical deficit. Pathological data are lacking, but quantitative magnetic resonance techniques combined with gadolinium-DTPA enhancement might help to establish whether this is so. The implication of the differences in the pattern of lesions between primary progressive disease and the other forms of multiple sclerosis is that important differences may exist in the pathogenesis of the lesions. Recent evidence obtained by using Gd-DTPA enhancement supports this possibility, ${ }^{14} 17$ as does some immunogenetic evidence. ${ }^{18}$

Our observations have important practical implications. Some of the lesions in patients with primary progressive multiple sclerosis are so small and insignificant that they can be easily missed; since completing this study we have seen several cases in which a scan was initially reported as normal but on careful review a small number of minute lesions were seen. If there are also differences in pathogenesis, including cases of primary progressive disease in a therapeutic trial in relapsing and remitting multiple sclerosis may obscure a beneficial effect.

In summary, no clear relation seems to exist between the abnormalities seen on magnetic resonance imaging and the disability experienced by patients with multiple sclerosis. Use of magnetic resonance imaging continues to increase our knowledge of the disease, but its role in management needs to be carefully defined if scarce resources are not to be wasted. It is, however, invaluable in diagnosing the disease. ${ }^{19}$

The Multiple Sclerosis NMR Research Group is supported by the Multiple Sclerosis Society of Great Britain and Northern Ireland and the Medical Research Council of Great Britain. AJT is supported by the Scarfe Trust. We thank Rob Clarke, of the computing and statistics unit, for his advice. 
1 Mcalpine D, Compston N. Some aspects of the natural history of disseminated sclerosis: incidence, course and prognosis; factors affecting onset and coursc. Q. J. Med 1952;21:135-67.

2 Confavreux C, Aimard G, Devic M. Course and prognosis of multiple sclerosis assessed by the computerized data processing of 349 patients. Brain 1980;103:281-300.

3 Weinshenker BG, Bass B, Rice GPA, et al. The natural history of multiple sclerosis: a geographical based study. 1. Clinical course and disability. Brain 1989:112:133-46

4 McAlpine D. The benign form of multiple sclerosis. A study based on 241 cases seen within three years of onset and followed up until the tenth year or cases seen within three years of onset and fol
more of the disease. Brain 1961;84:186-203.

5 Thompson AJ, Hutchinson M, Brazil J, et al. A clinical and laboratory studv of benign multiple sclerosis. Q F Med 1986;58:69-80.

6 McAlpine D. Course and prognosis in multiple sclerosis. In: McAlpine D, Lumsden CE, Acheson ED, eds. Multiple sclerosis: a reappraisal. Edinburgh Churchill Livingstone, 1972:206-7.

McDonald WI. Pathophysiology of multiple sclerosis. In: McDonald WI Silberberg DH, eds. Multiple sclerosis. London: Butterworths, 1986:128-9.

8 Stewart WA, Hall LD, Berry K, Paty DW. Correlation between NMR scan and brain slice data in multiple sclerosis. Lancet 1984;ii:412.

9 Ormerod IEC, Miller DH, McDonald WI, $t$ t al. The role of NMR imaging in the assessment of multiple sclerosis and isolated neurological lesions: quantitative study. Brain 1987:110:1579-616.
10 Poser CM, Paty DW, Schcinberg L, et al. New diagnostic criteria tor multiple sclerosis: guidetines for research protocols. Ann Neurol 1983;13:227-31.

11 Kurtzke JF. Rating neurologic impairment in multiple sclerosis: an expanded disability status scale (EDSS). Neurologv 1983:33:14+4-52.

12 Miller DH, Rudge P, Johnson (i, et al. Serial gadolinium enhanced magnetic resonance imaging in multiple sclerosis. Brain 1988;111:927-39.

13 Isaac C, L.i DKB, Genton M, et al. Multiple sclerosis: a serial study using MRI in relapsing patients. Neurologv 1988;38:1511-5.

14 Thompson AJ, Kermode AG, MacManus DG, Kingsley DPE, Moseles IF, MICDonald WI. Pathogenesis of progressive multiple sclerosis. Lance 1989;i:1322-3.

15 Koopmans RA, Li DKB, Grochowski E, Cutler PJ, Paty DW. Benign versus chronic progressive multiple sclerosis: magnetic resonance imaging features. Ann Neurol 1989;25:74-8

16 Kermode AG, Tofts PS, Thompson AJ, et al. Heterogeneity of blood-brain barrier changes in multiple sclerosis: an MRI study. Neurology (in press).

17 Kappos L, Stadt D, Rohrbach E, Keil W. Gadolinium-D'TPA enhanced magnetic resonance imaging in the evaluation of different discasc courses and discase activity in MS. Neurologv 1988;38 (Suppl 1):255.

18 Olerup (), Fredrikson S, Olsson T, Kam-Hansen H. HLA class II genes in chronic progressive and in relapsing/remitting multiple sclerosis. Lance 1987 ;ii:327.

19 McDonald WI. The diagnosis of multiple sclerosis. Br. Med f 1989:299:635-7.

\section{Social influences and cardiovascular risk factors as determinants of plasma fibrinogen concentration in a general population sample of middle aged men}

Annika Rosengren, Lars Wilhelmsen, Lennart Welin, Alecka Tsipogianni, Ann-Catrin Teger-Nilsson, Hans Wedel

Abstract
Objective-To analyse the relation between fibrinogen concentration and social class and other social factors found to be related to mortality. The results regarding cardiovascular disease are unpublished, as yet.

Design-Cross sectional population study.

Setting-City of Gothenburg, Sweden.

Subjects -639 Men from a population sample of 1016 men aged 50 in 1983. They were all employed and had no history of myocardial infarction or stroke. Fibrinogen values were available for all of them.

Main outcome measure-Fibrinogen concentration in relation to socioeconomic state according to occupation, and other social influences determined as number of people in the household and scores of social activities and activities in and outside the house.

Results-Men with low scores for activities at home had a mean plasma fibrinogen concentration of $3.34 \mathrm{~g} / 1$ (95\% confidence interval 3.21 to 3.47$)$, whereas those with an intermediate score had a mean concentration of $3.16(3.00$ to 3.32$) \mathrm{g} / 1$ and those with a high score $3.02(2.95$ to 3.10$) \mathrm{g} / \mathrm{l}$. Similar inverse relations were noted for the two other activity scores and for occupational class (class 1 being unskilled and semiskilled workers and class 5 professionals and executives) and the number of people in the household. Smoking exerted a strong influence on fibrinogen concentration, the relations between fibrinogen concentration and social factors being evident only in non-smokers. The mean difference in fibrinogen value between the non-smokers with the lowest activity scores at home and those with the highest scores was $0.36(0.19$ to 0.54$) \mathrm{g} / \mathrm{l}$, and similar differences were seen for the two other activity scores. Multiple regression analyses showed smoking, body mass index, the sum of all activities (inverse relation), and diabetes to be independently associated with fibrinogen value, whereas occupational class $(p=0.81)$ and the number of people in the household $(p=0.09)$ were not.
Conclusions-Psychosocial influences secm te influence the coagulation system in the body in a way that is associated with cardiovascular disease and premature death.

\section{Introduction}

High plasma concentrations of fibrinogen have been found to be independently associated with increased risk of cardiovascular disease in longitudinal studies.' Fibrinogen has also been implicated in the inverse relation between social class and coronary heart discase that has been described in Sweden ${ }^{6}$ and other Western industrialised countries. ${ }^{78}$ Social class is, however, associated with a wide variety of other psychosocial variables, such as measures of social support and network indices. Job stress was found to be related to fibrinogen concentration and contributed substantially to the differences found in fibrinogen concentration between different occupational grades. ${ }^{5}$ We examined the relation between fibrinogen concentration and occupational class and other measures of social influences that were previously found to be related to mortality in another sample of middle aged men. ${ }^{9}$

\section{Subjects and methods}

STUDY POPULATION

In 1983 a random sample was drawn of half of all the men in Gothenburg who were born in 1933. The men in the sample $(n=1016)$, all of whom were aged 50 , were invited to a health examination. In all, $776 \mathrm{men}$, or $76 \%$ of the sample, responded.

\section{EXAMINATIONS}

All examinations were performed in the morning. Blood pressure was measured with a mercury sphygmomanometer in the sitting position after five minutes' rest. Body weight was measured on a lever balance to the nearest $0 \cdot 1 \mathrm{~kg}$. Body mass index was calculated as weight in $\mathrm{kg} /$ (height in metres)?

To determine plasma fibrinogen concentrations 